\begin{tabular}{|c|l|}
\hline Title & The application of an InGaA s/GaA sN strain-compensated superlattice to InA squantum dots \\
\hline Author(s) & Zhang, Wei; Uesugi, Katsuhiro; Suemune, Ikuo \\
\hline Citation & Journal of A pplied Physics, 99(10), 103103-1-103103-7 \\
\hline https://doi.org/10.1063/2197261 \\
\hline Issue Date & 2006-05-15 \\
\hline Doc URL & http://hdl.handle.net/2115/14412 \\
\hline Rights & Copyright $\odot$ 2006 A merican Institute of Physics \\
\hline Type & article \\
\hline File Information & JA pplPhys_v99a103103.pdf \\
\hline
\end{tabular}

Instructions for use 


\title{
The application of an InGaAs/GaAsN strain-compensated superlattice to InAs quantum dots
}

\author{
Wei Zhang, Katsuhiro Uesugi, and Ikuo Suemune ${ }^{a)}$ \\ Research Institute for Electronic Science, Hokkaido University and CREST, JST, Kita-21, Nishi-10, \\ Sapporo 001-0021, Japan
}

(Received 12 October 2005; accepted 22 March 2006; published online 24 May 2006)

\begin{abstract}
Application of InGaAs/GaAsN strain-compensated superlattice (SCSL) to InAs quantum dots (QDs) has been studied with atomic force microscopy (AFM), reflection high-energy electron diffraction (RHEED), and temperature-dependent photoluminescence (PL) measurements. The insertion of a tensile-strained GaAsN layer between InGaAs layers with high In concentrations can compensate the compressive strain in the InGaAs layers and reduce the flattening of QDs during the growth of the successive InGaAs layers. Compared with QDs capped with a single InGaAs layer of a high In concentration, QDs capped with such SCSLs can achieve almost the same redshift of emission wavelength, while the optical property is highly improved. The mechanism responsible for this is discussed based on the AFM, RHEED, and PL measurements. (C) 2006 American Institute of Physics. [DOI: 10.1063/1.2197261]
\end{abstract}

\section{INTRODUCTION}

Quantum dots (QDs) have been attracting intensive attention in basic research and device applications due to their specific three-dimensional confinement. For example, longwavelength QDs offer excellent active regions for highperformance optical devices in the optical-fiber communication window. For InAs QDs grown on GaAs substrates, use of an InGaAs barrier reduces in principle the interface mismatch and is an effective way to extend the emission wavelength. Especially when the In composition is increased to $40 \%$, redshift up to the wavelength of $1.5 \mu \mathrm{m}$ has been reported. ${ }^{1}$

However, the optical quality of QDs capped with such In-rich InGaAs layers shows serious degradation. This results from the highly accumulated compressive strain in both the dots and barriers. It is well known that dislocation generation takes place when the accumulated total compressive strain exceeds a critical value, and it has been argued that these dislocations induce diffusion of In atoms from InGaAs layers and also from already formed InAs QDs. This was identified by a decrease of dot sizes. ${ }^{2,3}$ Therefore, it is important to reduce the excessive compressive strain in the InGaAs barrier layers.

It has been reported that a tensile-strained GaAsN layer can compensate a part of the compressive strain induced in the QDs, and the application of the GaAsN barrier layers lead to the improved luminescence intensity of the QDs. ${ }^{4,5}$ Meanwhile, superlattice (SL) structures are frequently used as buffer layers in optoelectronic devices to improve the crystalline quality by capturing defects at heterointerfaces. Based on the above considerations, we proposed an application of InGaAs/GaAsN strain-compensated superlattice (SCSL) as a barrier layer of InAs QDs. Each pair of such SCSLs consists of a thin compressively strained InGaAs layer with a high In concentration and a thin tensile-strained

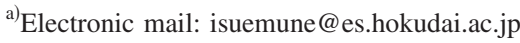

GaAsN layer. The GaAsN layer will compensate the overall strain in the system while keeping the presence of the heterointerface with reduced lattice mismatch between the InGaAs barrier and the InAs QDs. Properties of the QDs capped with such InGaAs/GaAsN SCSL are discussed with atomic force microscopy (AFM), reflection high-energy electron diffraction (RHEED), and temperature-dependent photoluminescence (PL) measurements. Underlying mechanisms of such SCSL are discussed.

\section{EXPERIMENTAL PROCEDURE}

QDs in this work were grown on epiready (001) semiinsulating (SI) GaAs substrates with a metal-organic (MO) molecular-beam epitaxy (MBE) system. The growth procedure is as follows. After substrate surface cleaning at $550{ }^{\circ} \mathrm{C}$, a 100 -nm-thick GaAs buffer layer was grown, and then the temperature was decreased to $480{ }^{\circ} \mathrm{C}$. InAs QDs were formed with a growth rate of 0.0086 monolayer (ML)/s. The formation of InAs QDs was identified by the change of the RHEED patterns from streaky to spotty ones. After the spotty RHEED pattern appeared, the growth of InAs was stopped immediately but the As supply was continued for $20 \mathrm{~s}$. This increased the specular-spot RHEED intensity to a saturated level and this will suggest the completion of adatom surface migration. Then the As supply was stopped and the substrate temperature was decreased to $450{ }^{\circ} \mathrm{C}$ with the ramping rate of $30^{\circ} \mathrm{C} / \mathrm{min}$. The spotty RHEED pattern did not show any clear change during this cooling process. The growth condition of the InAs QDs was kept constant in this work. The average dot height and diameter are 7.1 and $38 \mathrm{~nm}$, respectively. Sometimes there are small fluctuations $(\sim 7 \%)$ in dot sizes among samples grown in different days. Then capping layers such as a single InGaAs layer, a single GaAs layer, an InGaAs/GaAs SL, and an InGaAs/GaAsN SCSL were grown for each sample. All the samples were completed with the growth of additional 30-nm-thick GaAs barrier layers. Here, we adopted the lower growth tempera- 
ture of $450{ }^{\circ} \mathrm{C}$ to grow cap and barrier layers to reduce the indium segregation during the capping process, which is monitored by a RHEED equipment.

The MO precursors employed for $\mathrm{Ga}$, In, As, and $\mathrm{N}$ are triethylgallium (TEGa), triethylindium (TEIn), trisdimethylaminoarsenic (TDMAAs), and monomethylhydrazine (MMHy), respectively. The In and $\mathrm{N}$ compositions in InGaAs and GaAsN are $37.5 \%$ and $2.2 \%$, respectively, which were determined by high-resolution $\mathrm{x}$-ray diffraction measurements. The In and $\mathrm{N}$ concentrations in the InGaAs and GaAsN layers, respectively, were kept constant in this paper.

The PL measurements were carried out using the secondharmonic generation of a yttrium aluminum garnet (YAG) laser at the wavelength of $532 \mathrm{~nm}$. The sample temperature was changed from 20 to $300 \mathrm{~K}$ in a closed-cycle helium cryostat. An InGaAs detector was used to collect the signal dispersed by a $0.5-\mathrm{m}$ monochromator via lock-in mode detection scheme.

\section{RESULTS AND DISCUSSIONS}

Firstly, the temperature dependence of ground-state (GS) PL peak wavelengths and PL integrated intensities of the QDs samples was examined, and the results are shown in Figs. 1(a) and 1(b), respectively. The difference of the sample structures is the initial capping layer with the common 30-nm-thick GaAs barrier layer. The details of the capping layer in each sample are as follows: InGaAs/GaAs SL, InGaAs/GaAsN SCSL, 1.6-nm-thick single InGaAs layer, and 6-nm-thick pure GaAs layer. For the two SL structures, the thicknesses of InGaAs and $\mathrm{GaAs}(\mathrm{GaAsN})$ are 1.1 and $2 \mathrm{~nm}$, respectively. The pair number is 2 . The total thickness of the SLs is therefore $6.2 \mathrm{~nm}$. The average residual strain in the capping layer can be calculated by $\varepsilon_{\mathrm{av}}=\left(\varepsilon_{\mathrm{GaAsN}} L_{\mathrm{GaAsN}} m_{\mathrm{GaAsN}}+\varepsilon_{\mathrm{InGaAs}} L_{\mathrm{InGaAs}} m_{\mathrm{InGaAs}}\right) /$ $\left(L_{\mathrm{GaAsN}} m_{\mathrm{GaAsN}}+L_{\mathrm{InGaAs}} m_{\mathrm{InGaAs}}\right)$, where $\varepsilon_{\mathrm{GaAsN}}(-0.45 \%)$ and $\varepsilon_{\text {InGaAs }}(2.65 \%)$ are biaxial strains of the GaAsN and InGaAs layers, respectively. $L_{\mathrm{GaAsN}}$ and $L_{\mathrm{InGaAs}}$ are the corresponding thicknesses of the GaAsN and InGaAs layers, respectively. $m_{\mathrm{GaAsN}}$ and $m_{\mathrm{InGaAs}}$ are the numbers of the GaAsN and InGaAs layers, respectively. In the above case, $0.65 \%$ is the average residual strain for InGaAs/GaAsN SCSLs; $0.94 \%$ is the average residual strain for GaAs/InGaAs SLs.

The sample with pure GaAs capping and barrier layers shows the shortest emission wavelength but the strongest optical intensity. The emission wavelengths at 20 and $300 \mathrm{~K}$ are 1155 and $1245 \mathrm{~nm}$, respectively. The QDs capped with 1.6-nm-thick InGaAs exhibit the longest wavelength but the poorest optical quality, which is consistent with the previously reported general characteristics of QDs capped with high-In InGaAs. ${ }^{1,7}$ Under the current growth parameters, no emissions were observed from the QDs capped with single InGaAs layers the thickness of which exceeds $2 \mathrm{~nm}$.

The main finding comes from the difference of the samples capped with two kinds of SL structures. It will be clear from Fig. 1 that the QDs capped with the InGaAs/GaAsN SCSL show longer emission wavelength extending to the $1.3-\mu \mathrm{m}$ optical-fiber communication wavelength and improved PL integrated intensity at room tem-
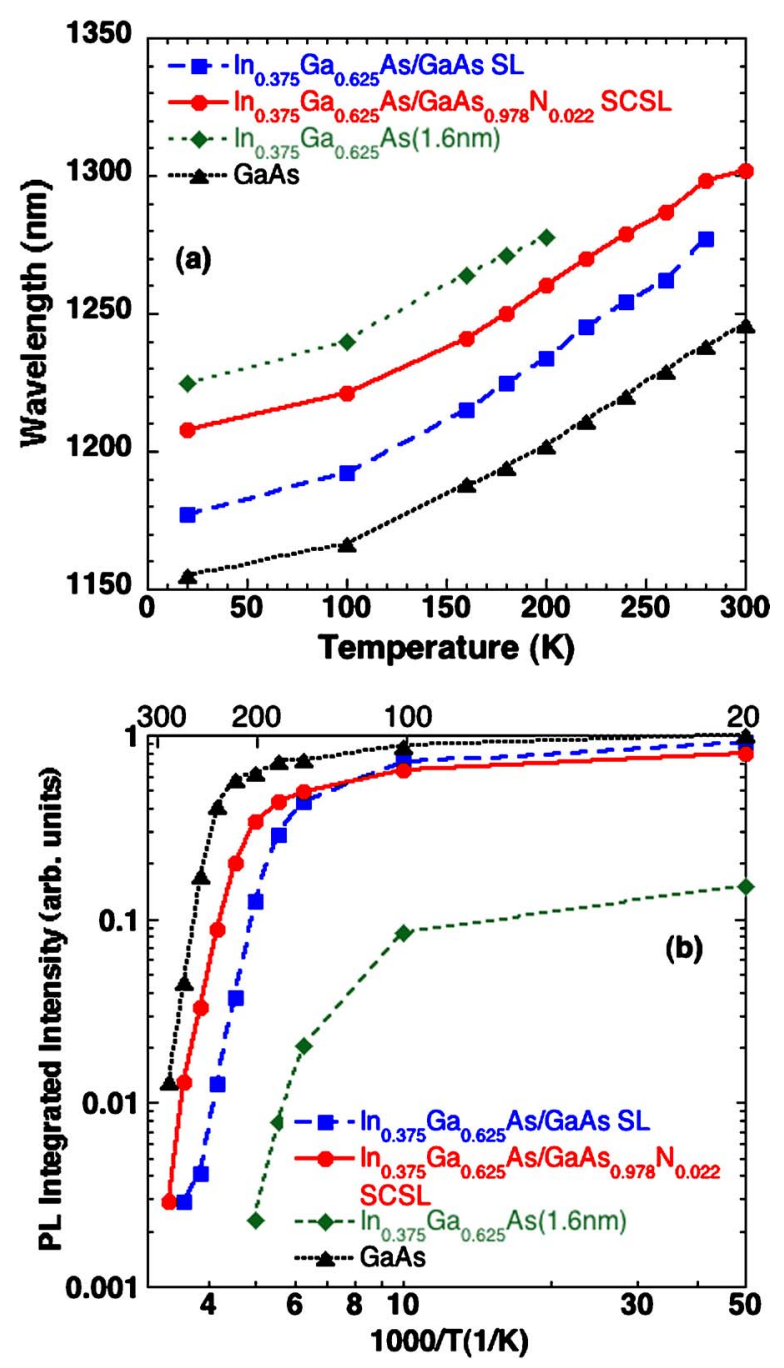

FIG. 1. (Color online) (a) and (b) are temperature-dependent wavelengths and PL integrated intensities of the QDs capped with two pairs of InGaAs/GaAs SLs, two pairs of InGaAs/GaAsN SCSLs, 1.6-nm-thick InGaAs, and pure GaAs, respectively. The thicknesses of InGaAs and $\mathrm{GaAs}(\mathrm{N})$ in the SLs are 1.1 and $2 \mathrm{~nm}$, respectively.

perature by about five times compared with the QDs capped with the InGaAs/GaAs SL. This demonstrates that the insertion of the tensile-strained thin GaAsN layers is effective to modify the optical properties. The difference of the two cases will be discussed in more detail below.

Figures 2(a) and 2(b) are the PL spectra measured on these samples with two kinds of SL capping structures at 20 and $100 \mathrm{~K}$, respectively. In addition to the PL peaks originated from the GS transitions in QDs designated as sp1, the subpeaks from the SL capping layers designated as sp2 were observed. The solid line is the spectrum observed from the InAs QDs capped with the InGaAs/GaAsN SCSL and the sp1 peak showed redshift compared with the sp1 peak observed from the one capped with the InGaAs/GaAs SL. In contrast, the sp2 peak observed from the InGaAs/GaAsN SCSL shown by the solid line showed blueshift compared with the one observed from the InGaAs/GaAs SL. In the present two SLs, the difference, in principle, is the replacement of the GaAs layers with the GaAsN layers. Since GaAsN has the large band gap bowing, the energy gap of 

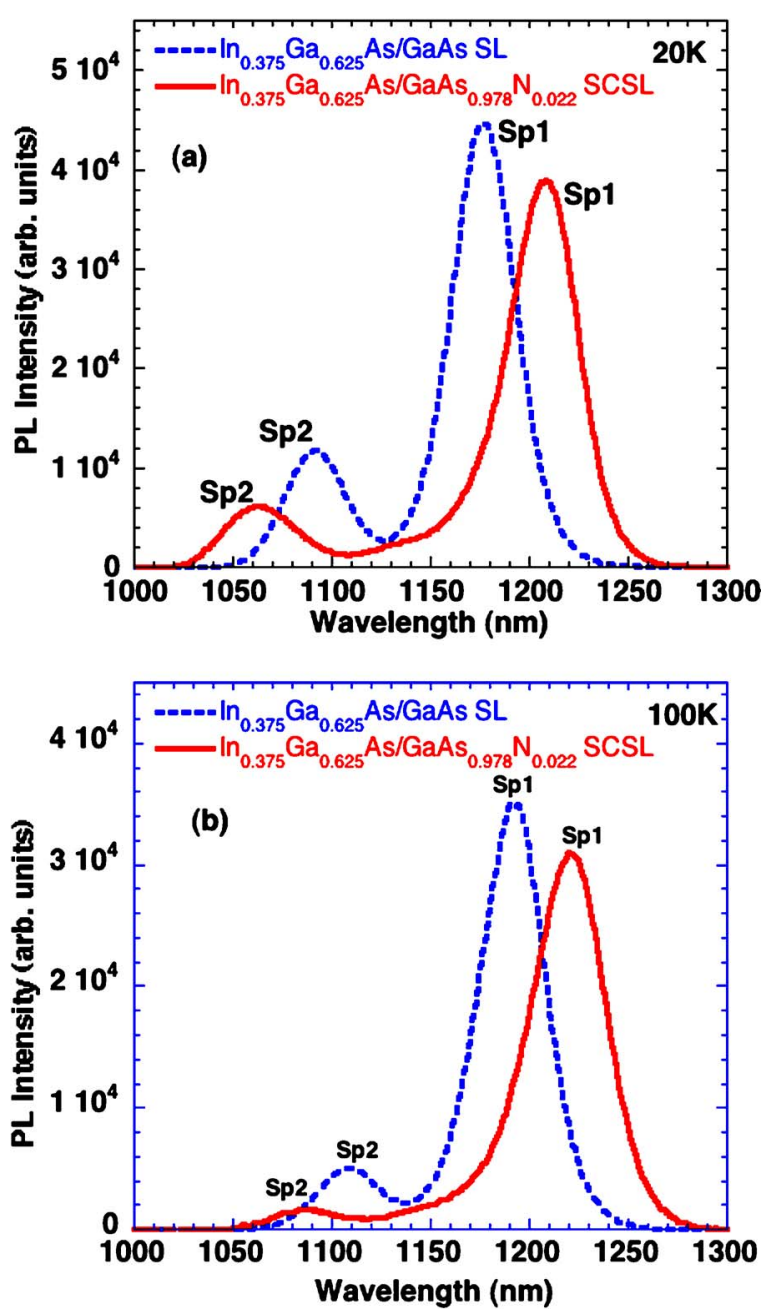

FIG. 2. (Color online) PL spectra of QDs capped with two pairs of InGaAs/GaAs SLs and QDs capped with two pairs of InGaAs/GaAsN SCSLs. (a) 20 and (b) $100 \mathrm{~K}$. The thicknesses of InGaAs and GaAs(N) are 1.1 and $2 \mathrm{~nm}$, respectively.

GaAsN will be reduced by $0.26 \mathrm{eV}$ from that of GaAs for the $\mathrm{N}$ concentration of $2.2 \%{ }^{8}$ Meanwhile, the band discontinuity is mostly localized to the conduction band in this combination, the band offset at the InGaAs/GaAsN interface will coordinately be reduced compared with that at the InGaAs/GaAs interface. Therefore the redshift of the sp2 peak observed with the InGaAs/GaAs SL relative to that observed with the InGaAs/GaAsN SCSL cannot be accounted for without considering the increase of the In concentration in the InGaAs layer in the InGaAs/GaAs SL. The relations of the sp1 and sp2 peak energies in the two samples with the two kinds of SL will be discussed in more detail in the following based on the additional AFM and RHEED observations.

In order to clarify the difference of the roles between the GaAs and GaAsN layers in the SLs, the growth process of the SL was examined by the observations of the RHEED patterns during the growth. Figure 3(a) shows the RHEED pattern observed in the [110] direction just after the formation of InAs QDs. The clear shift from streaky to spotty RHEED pattern shown in Fig. 3(a) indicates the StranskiKrastanow (SK) growth of the QDs. After the formation of
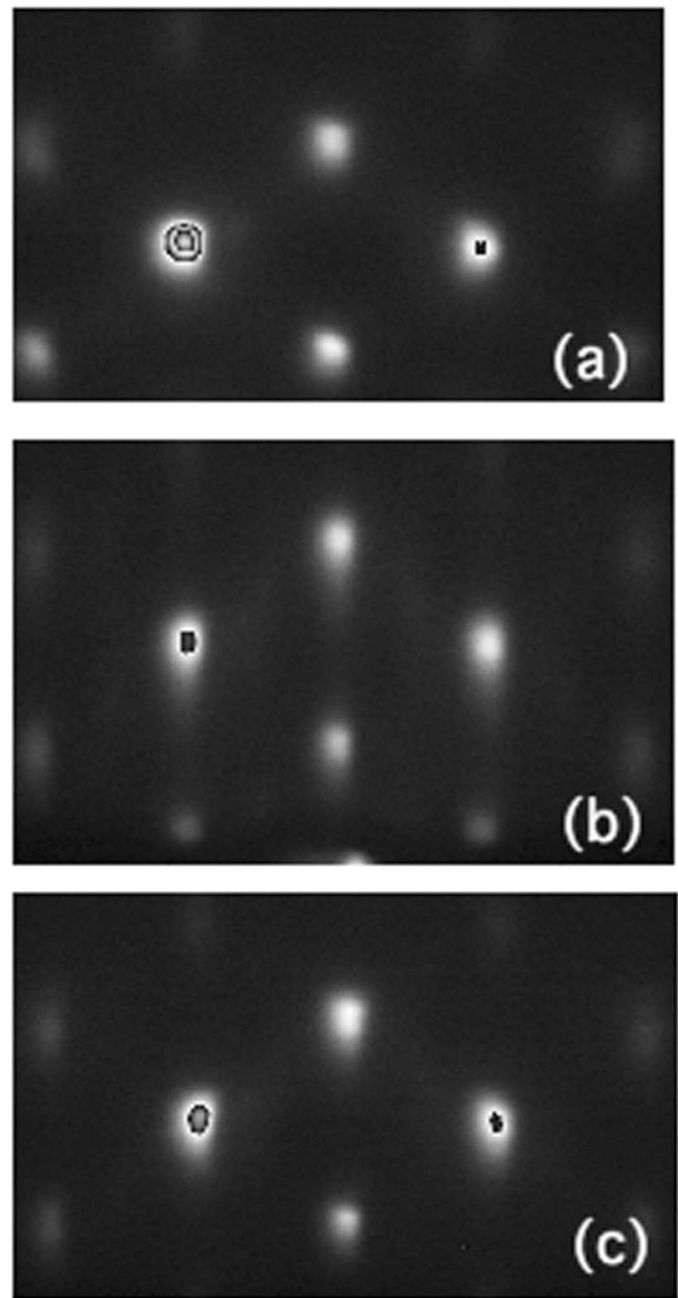

FIG. 3. (Color online) RHEED patterns observed in the [110] direction for (a) bare QDs, (b) QDs capped with InGaAs/GaAs/InGaAs, and (c) QDs capped with InGaAs/GaAsN/InGaAs. The thicknesses of InGaAs and $\operatorname{GaAs}(\mathrm{N})$ are 1.1 and $2 \mathrm{~nm}$, respectively.

InAs QDs, the two kinds of SLs were formed. Figure 3(b) is the RHEED pattern observed after the formation of three stacks of 1.1-nm-thick InGaAs/2-nm-thick GaAs/1.1-nm-thick InGaAs layers. Although the total layer thickness of $4.2 \mathrm{~nm}$ is thinner than the bare QD height of $7.1 \mathrm{~nm}$, the spotty RHEED pattern was superimposed with the streaky one elongated along the [110] direction as shown in Fig. 3(b). Although it is not clear in Fig. 3(b), weak $\times 2$ reconstruction also appeared, which means the flattening of the surface. When the RHEED measurement was performed after the formation of 1.1-nm-thick InGaAs/2-nm-thick GaAsN/1.1-nm-thick InGaAs layers on the InAs QDs, the spotty RHEED pattern shown in Fig. 3(c) was observed, which is almost similar to that in Fig. 3(a). This difference suggests that the deformation of the InAs QDs takes place during the capping process with the InGaAs/GaAs layers. Therefore the sample surfaces corresponding to Figs. 3(b) and 3(c) were examined with AFM observations.

Figures 4(a)-4(c) are the AFM images of bare QD surface corresponding to Fig. 3(a) and the QD surfaces after the formation of the second InGaAs layer corresponding to Figs. $3(\mathrm{~b})$ and 3(c), respectively. The average diameter and height 

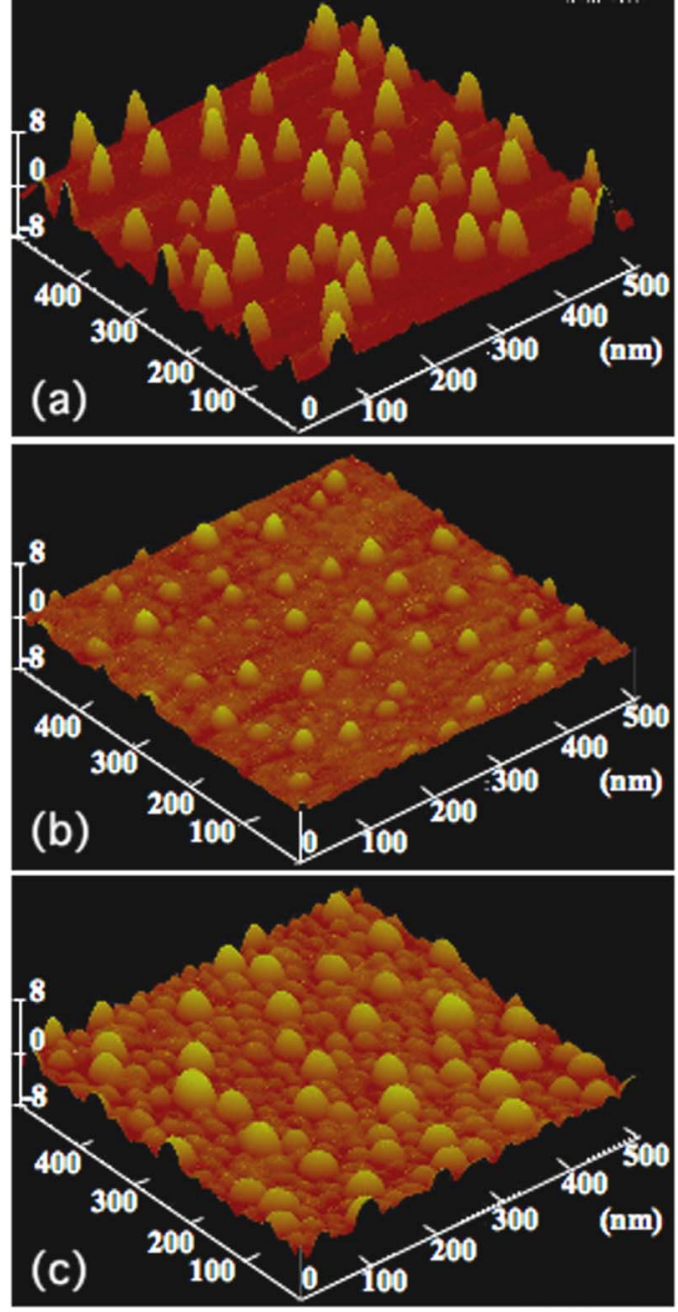

FIG. 4. (Color online) $500 \times 500 \mathrm{~nm}^{2}$ AFM images for (a) bare QDs, (b) QDs capped with $\operatorname{InGaAs}(1.1 \mathrm{~nm}) / \operatorname{GaAs}(2 \mathrm{~nm}) / \operatorname{InGaAs}(1.1 \mathrm{~nm})$, and (c) QDs capped with $\operatorname{InGaAs}(1.1 \mathrm{~nm}) / \mathrm{GaAsN}(2 \mathrm{~nm}) / \operatorname{InGaAs}(1.1 \mathrm{~nm})$.

of the bare dots shown in Fig. 4(a) are 22 and $7.1 \mathrm{~nm}$, respectively. After the formation of the InGaAs/GaAs/InGaAs layers with the total thickness of $4.2 \mathrm{~nm}$, the mean dot height observed on the sample surface was reduced as shown in Fig. 4(b) and was $2.1 \mathrm{~nm}$ in height. Since the formation of the three stacks of layers on the InAs QDs will result in the apparent average dot heights higher than $2.9 \mathrm{~nm}$, the observation of the reduced dot heights suggests the deformation of the InAs QDs during the capping process. Similar AFM observation on the sample capped with the InGaAs/GaAsN/InGaAs layers is shown in Fig. 4(c), and the surface dot structures showed the average dot size of $20.5 \mathrm{~nm}$ in diameter and $3.1 \mathrm{~nm}$ in height, the height of which is almost equal to the difference of the average bare dot height and the three-stacked layer thickness.

Figure 5 shows the schematic change from (a) bare dots to (b) QDs capped with InGaAs/GaAs/InGaAs and (c) QDs capped with $\mathrm{InGaAs} / \mathrm{GaAsN} / \mathrm{InGaAs}$. From the above AFM observations, the InAs QDs capped with the InGaAs/GaAs/InGaAs layers showed distinct reduction of the dot height by $\sim 1 \mathrm{~nm}$ in average during the capping process as shown in Fig. 5(b), although there is some extension in the base diameter than the ideal one with the difference

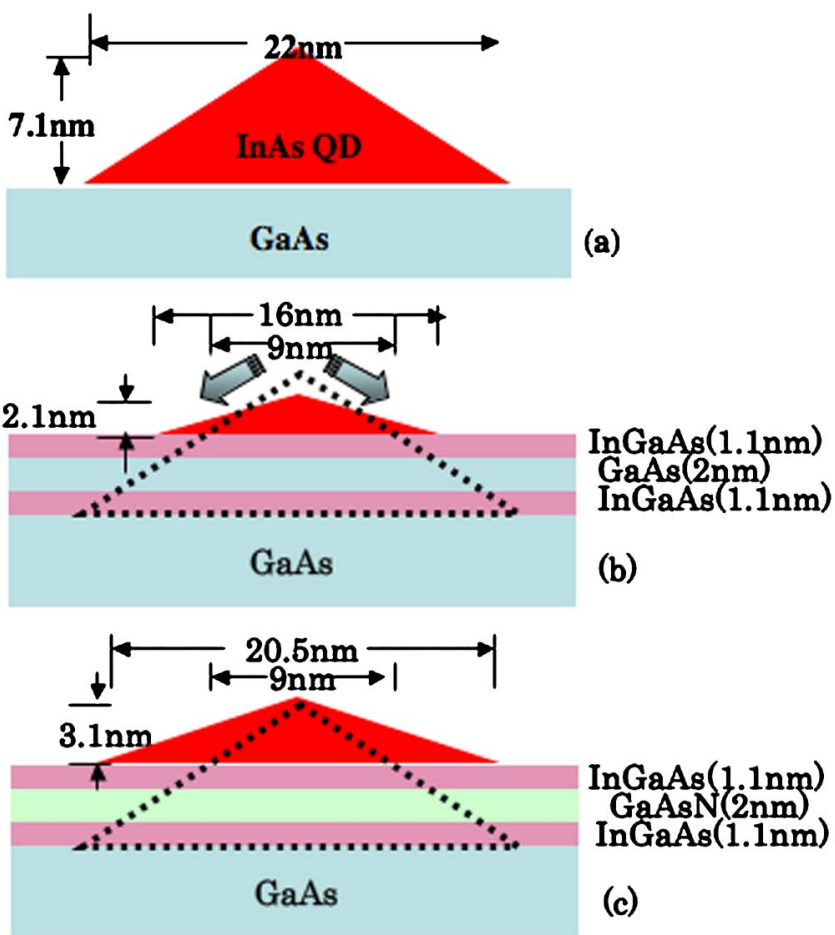

FIG. 5. (Color online) Schematic illustration of (a) bare InAs QD, (b) InAs QD capped with InGaAs/GaAs/InGaAs, and (c) InAs QD capped with InGaAs/GaAsN/InGaAs.

between the bare dot and capping layer thickness. The situation was much different when the GaAs layer was replaced with the GaAsN layer. The distinct reduction of the dot height during the capping process is not observed in this case, which is shown in Fig. 5(c). The extension of the dot diameter was also observed in this case, which may be due to the overgrowth of the capping layers on the dot side facets.

The revealed difference of the dot size deformations during the capping processes with these two kinds of structures shows that the tensile-strained GaAsN layers help to preserve the QD shape. The lateral In diffusion from the InAs QDs to the capping layers will coordinately be reduced by the insertion of the GaAsN layers. Therefore, the relative relations of the sp1 and sp2 peaks in Fig. 2 are interpreted by the difference of the lateral In diffusion. That is, the lateral In diffusion from InAs QDs to InGaAs/GaAs capping layers is more enhanced for the sample capped with the InGaAs/GaAs/InGaAs stacked layers in comparison to the sample capped with the GaAsN related stacked layers. The resultant smaller-sized InAs QDs in the former sample will show the relative blueshift of the sp1 peak, while the diffusion-induced increase of In concentrations especially in the InGaAs capping layers will show the relative redshift of the sp2 peak as observed in Fig. 2.

It has been studied with transmission electron microscopy (TEM) that in usual InAs/GaAs QDs, the redistribution of In atoms on the surface of QDs will finally determine the buried QD size through segregation, intermixing, and lateral diffusion with capping layers. ${ }^{9}$ These dynamical processes depend strongly on the surface In atom density and the resultant strain field between dots and capping layers. So the present experimental results show that the insertion of the 
tensile-strained GaAsN serves to stabilize the dynamical behavior of the surface In atoms through the modification of the strain field.

The underlying physics about how the GaAsN layers affect the surface dynamical process is not conclusive at present, but two interactive potential mechanisms will be pointed out. (i) SC effect. It has been reported that in the GaAsN capped InAs QDs, the strain fields around the dots are substantially reduced and the insertion of a thin tensilestrained GaAsN compensates a part of the total strain. ${ }^{10,11}$ The reduction of the strain field decreases the segregation, intermixing, and diffusion during the successive capping process. (ii) Local strain configuration: A valence force field (VFF) model calculation shows that In atoms on the QD surface capped with GaAs are under the outward stress, while nitrogen $(\mathrm{N})$ one-monolayer coverage on the QD surface changes the direction of stress field on In atoms toward inward. This will help to suppress the In segregation from the InGaAs layers with high In concentrations or from InAs QDs with the presence of $\mathrm{N}$ atoms at the interfaces. ${ }^{12,13}$

The thermal activation energy $E_{A}$ of the temperaturedependent luminescence intensity is estimated by fitting the temperature-dependent PL integrated intensity $I_{\mathrm{PL}}(T)$ with a thermal activation mechanism formula, ${ }^{14} I_{\mathrm{PL}}(T)$ $=C /\left[1+A \exp \left(-E_{A} / k_{B} T\right)+B\right]$, where $A, B$, and $C$ are fitting parameters, $k_{B}$ is the Boltzmann constant, and $T$ is the sample temperature. The fitted activation energies for the four kinds of QDs capped with SL, SCSL, InGaAs, and GaAs are 248, 300, 200, and $310 \mathrm{meV}$, respectively. The energy separations between the sp1 and sp2 peaks shown in Fig. 2 for QD samples capped with InGaAs/GaAs SL and InGaAs/GaAsN SCSL are estimated to be 82 and $145 \mathrm{meV}$, respectively, which are much smaller than the measured thermal activation energies. The temperature dependence of the PL intensity ratio between the sp1 and sp2 peaks shown in Fig. 2 cannot be explained with the Boltzmann statistics and this indicates that carrier transfer between the QDs and SL capping layers is not efficient. This suggests that the luminescence quenching mechanism at the higher temperature is not necessarily the thermionic emission from QDs to the capping layers. The lower thermal activation energies measured on the QDs capped with the single InGaAs capping layer is consistent with the lower luminescence efficiency shown in Fig. 1(b) considering the generation of defectrelated deep levels in this sample, which efficiently quench luminescence.

It will be interesting to study the effect of the SL pair number on the emission wavelength of the InAs QDs. In this case, the thicknesses of the InGaAs and GaAsN layers were set to 1.1 and $1.1 \mathrm{~nm}$, respectively. The pair numbers studied were $1,2,3,4$, and 5 , where the corresponding thicknesses of the SCSLs are 2.2, 4.4, 6.6, 8.8, and $11 \mathrm{~nm}$, respectively. The variation of the [110] RHEED patterns observed on the sample surfaces with the 0 , one, two, three, four, and five pairs of SCSLs is shown in Figs. 6(a)-6(f). From the thickness of the SCSL, the QDs will be present on the grown surfaces up to the pair number of 2, and the spotty RHEED patterns were consistently observed in Figs. 6(a)-6(c). QDs will be almost covered with the pair number of 3 , where the
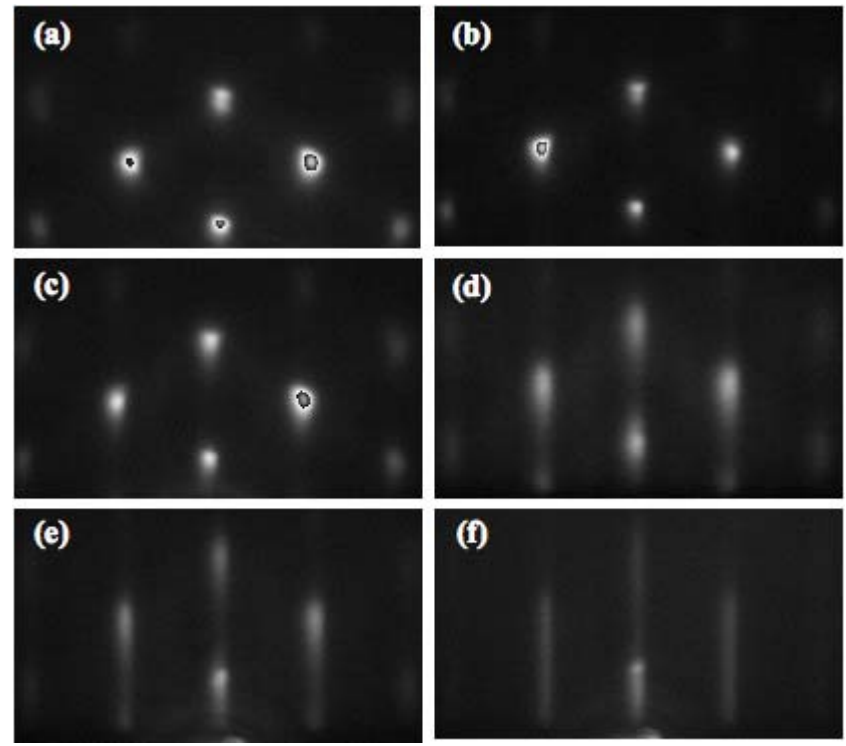

FIG. 6. (Color online) (a), (b), (c), (d), (e), and (f) are RHEED patterns of QDs capped with 0, one, two, three, four, and five pairs of InGaAs(1.1 nm)/GaAsN(1.1 nm) SCSLs observed in the [110] direction.

RHEED pattern shown in Fig. 6(d) is superimposed with the streaky one. Further increase of the SCSL pair numbers changed the RHEED pattern to more streaky as shown in Figs. 6(e) and 6(f), suggesting the recovery of atomically smooth surface after complete coverage of the QDs.

Figure 7 is the AFM measurements of surface dot size distributions of the samples covered with 0 , one, and two pairs of SCSLs. In this case, the mean diameters and the heights of the bare QDs, QDs buried with one pair of SCSL, and QDs buried with two pairs of SCSLs were 24 and $6.6 \mathrm{~nm}, 26$ and $4 \mathrm{~nm}$, and 19 and $1.6 \mathrm{~nm}$, respectively. The horizontal dashed lines in the figure are the expected height difference between the bare QD height and the buried SCSL thickness, assuming that the QD height does not change during the capping process. The measured dot size distributions

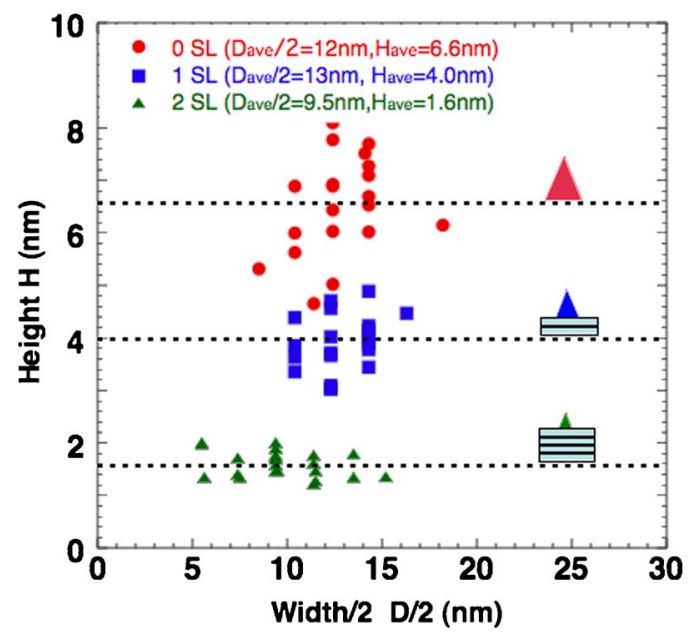

FIG. 7. (Color online) Dot size distribution of the QDs capped with 0, one, and two pairs of $\operatorname{InGaAs}(1.1 \mathrm{~nm}) / \mathrm{GaAsN}(1.1 \mathrm{~nm})$ SCSLs. The horizontal dashed lines in the figure are indicators of the ideal difference between the bare QD height and the SCSL thickness. The data in the brackets of the inset are the mean full width and height of the corresponding QDs. 

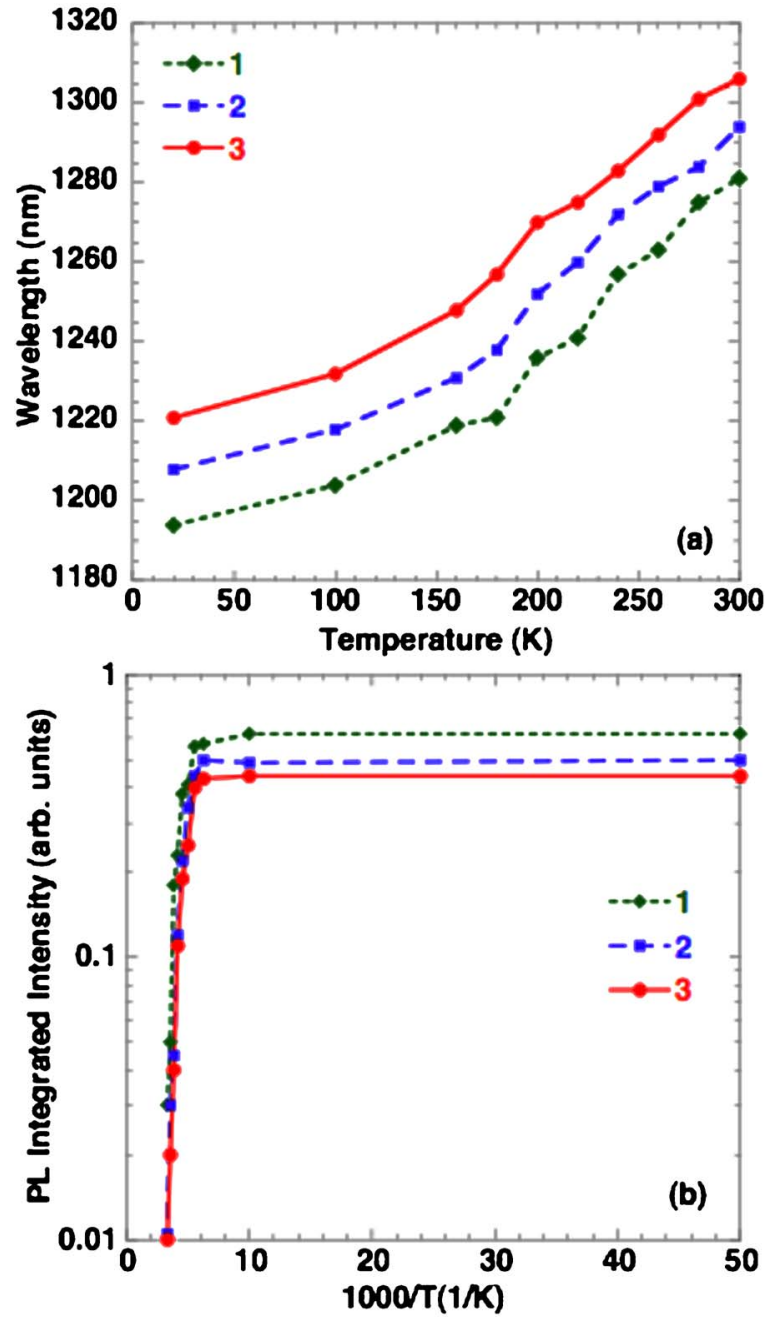

FIG. 8. (Color online) Temperature dependence of (a) PL wavelengths and (b) PL integrated intensities of the QDs capped with one, two, and three pairs of $\operatorname{InGaAs}(1.1 \mathrm{~nm}) / \mathrm{GaAsN}(1.1 \mathrm{~nm})$ SCSLs.

show the nice correspondence with the respective lines as shown in Fig. 7. Both the RHEED patterns shown in Fig. 6 and the AFM results confirm that the serious flattening of QDs does not occur when they are capped with the InGaAs/GaAsN SCSLs.

The temperature dependence of the GS peak wavelengths and the PL integrated intensities of the QDs capped with one, two, and three pairs of SCSLs are shown in Figs. 8(a) and 8(b), respectively. It will be clear in Fig. 8(a) that the PL peaks are redshifted with the increase of the pair number. Compared with the reference sample with the GaAs cap shown in Fig. 1(a), redshifts of the samples with the one, two, and three pairs of SCSLs at $20 \mathrm{~K}$ were 39,53 , and $66 \mathrm{~nm}$, respectively, and were almost parallel shifts regardless of the temperature. Further increase of the SCSL pair number from three to four resulted in a slight blueshift by $9 \mathrm{~nm}$. The luminescence intensities of the QDs were changed gradually from $73 \%, 61 \%$, and $54 \%$ relative to the reference sample, when the pair number was increased from one to three, respectively. Especially the sample capped with four pairs of SCSLs showed further degradation up to the relative luminescence intensity of $11 \%$. Both the observed blueshift of the emission wavelength and the accelerated degradation

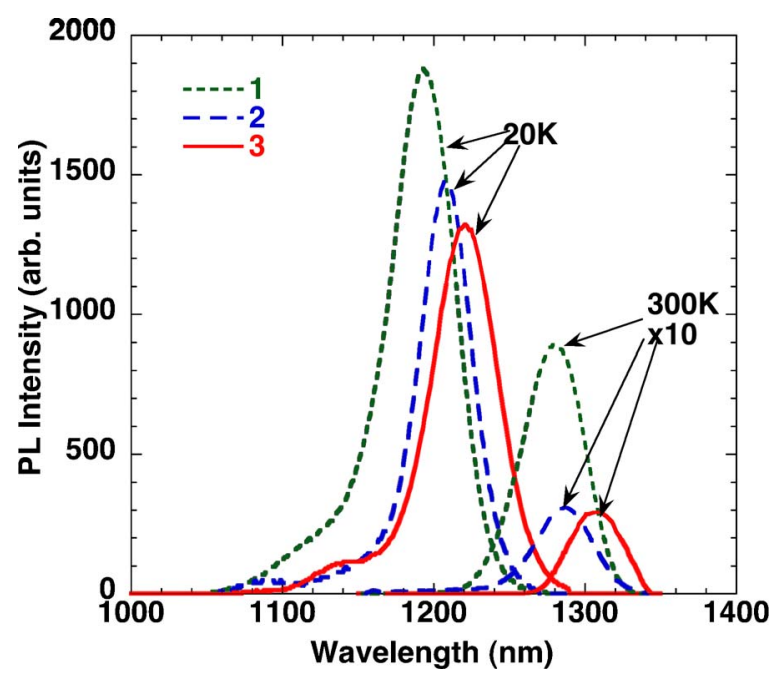

FIG. 9. (Color online) Low temperature $(20 \mathrm{~K})$ and room temperature (300 K) PL spectra of the samples capped with one, two, and three pairs of InGaAs(1.1 nm)/GaAsN(1.1 nm) SCSLs.

of the luminescence efficiency will suggest that the overall compressive strain within and around the QDs capped with the four pairs of SCSLs exceeds the critical strain accumulation. An exact estimation of the strain situation of the dot samples will be complex. But a simple estimation is about the average strain in the InGaAs/GaAsN SCSL, which is $2.2 \%$ in the present structures. The critical thickness estimated for the medium with the average strain will be $\sim 3.5 \mathrm{~nm}$ following Matthews and Blakeslee's theory. ${ }^{15}$ It is therefore possible that the present InAs QD samples capped with several pairs of SCSLs are under the influence of misfit defect generations. The employment of SCSL with the higher thickness ratio of the InGaAs/GaAsN layers with the development of a higher thickness control technique will improve the extension of the emission wavelength and the luminescence efficiency.

Figure 9 shows the PL spectra measured on the samples capped with one, two, and three pairs of SCSLs at 20 and $300 \mathrm{~K}$. The QDs capped with three pairs of SCSLs showed the redshift which is nearly of the same wavelength as that of the QDs capped with the 1.6-nm-thick $\operatorname{In}_{0.375} \mathrm{Ga}_{0.625} \mathrm{As}$ layer, while the luminescence efficiency was highly improved. Although the QDs capped with the 1.6-nm-thick $\mathrm{In}_{0.375} \mathrm{Ga}_{0.625}$ As layer showed serious degradation of the luminescence efficiency and the PL signal was quenched above $200 \mathrm{~K}$, clear luminescence at the wavelength of $1.3 \mu \mathrm{m}$ was observed from the QDs capped with three pairs of SCSLs at $300 \mathrm{~K}$.

Capping InAs QDs with the InGaAs/GaAsN SCSL or with a single InGaAs layer can be compared in another way. The average strain in the $\operatorname{In}_{0.375} \mathrm{Ga}_{0.625} \mathrm{As} / \mathrm{GaAs}_{0.978} \mathrm{~N}_{0.022}$ SCSL is $0.65 \%$. The In composition in an InGaAs layer corresponding to the $0.65 \%$ biaxial strain is $9 \%$. The redshift possible with this In composition in the InGaAs capping layer will be about $20 \mathrm{~nm}$ in the emission wavelength, ${ }^{1}$ which is much limited compared with the redshift of about $70 \mathrm{~nm}$ observed with the three periods of $\mathrm{In}_{0.375} \mathrm{Ga}_{0.625} \mathrm{As} / \mathrm{GaAs}_{0.978} \mathrm{~N}_{0.022}$ SCSL capping layer. This difference will be attributed to both the strain reducing 
effect ${ }^{1,7}$ of the $\operatorname{In}_{0.375} \mathrm{Ga}_{0.625} \mathrm{As}$ layer and the strain compensating effect ${ }^{4,10}$ of the $\mathrm{GaAs}_{0.978} \mathrm{~N}_{0.022}$ layer, which made the redshift approaching the $\operatorname{In}_{0.375} \mathrm{Ga}_{0.625}$ As capping layer possible in spite of the weak average strain of $0.65 \%$.

\section{CONCLUSIONS}

In summary, the application of an InGaAs/GaAsN SCSL to the InAs QDs was proposed. It was shown that the insertion of a tensile-strained GaAsN layer can suppress In diffusion out of QDs and help to prevent the flattening of the QDs during the growth of the successive InGaAs layer by compensating a part of the compressive strain of the former InGaAs layer. With proper selection of the SL parameters, InAs QDs capped with such InGaAs/GaAsN SCSLs can extend the emission to the $1.3-\mu \mathrm{m}$ optical-fiber communication wavelength, which is achievable with the QDs capped with single InGaAs barriers, but at the same time with much improved optical properties.

\section{ACKNOWLEDGMENTS}

This work was supported in part by Grant-in-Aid for Scientific Research on Priority Area (A), No. 17068001.
${ }^{1}$ J. Tatebayashi, M. Nishioka, and Y. Arakawa, Appl. Phys. Lett. 78, 3469 (2001).

${ }^{2}$ M. V. Maximov et al., Phys. Rev. B 62, 16671 (2000).

${ }^{3}$ F. Guffarth, R. Heitz, A. Schliwa, O. Stier, N. N. Ledentsov, A. R. Kovsh, V. M. Ustinov, and D. Bimberg, Phys. Rev. B 64, 085305 (2000).

${ }^{4}$ X. Q. Zhang, S. Ganapathy, I. Suemune, H. Kumano, K. Uesugi, Y. Nabetani, and T. Matsumoto, Appl. Phys. Lett. 83, 4524 (2003).

${ }^{5}$ O. Schumann et al., Phys. Rev. B 71, 245316 (2005).

${ }^{6}$ R. C. Tu, Y. K. Su, W. H. Lan, and F. R. Chien, J. Cryst. Growth 201-202, 961 (1999).

${ }^{7}$ H. Y. Liu et al., J. Appl. Phys. 88, 3392 (2000).

${ }^{8}$ I. Suemune, K. Uesugi, and W. Walukiewicz, Appl. Phys. Lett. 77, 3021 (2000).

${ }^{9}$ Q. Gong, P. Offermans, R. Nötzel, P. M. Koenraad, and J. H. Wolter, Appl. Phys. Lett. 85, 5697 (2004).

${ }^{10}$ S. Ganapathy, X. Q. Zhang, I. Suemune, K. Uesugi, H. Kumano, B.-J. Kim, and T.-Y. Seong, Jpn. J. Appl. Phys., Part 1 42, 5598 (2003).

${ }^{11}$ N. Matsumura, S. Muto, S. Ganapathy, I. Suemune, K. Numata, and K. Yabuta, Jpn. J. Appl. Phys., Part 2 45, L57 (2006).

${ }^{12}$ Y. Nabetani, T. Matsumoto, G. Sasikala, X. Q. Zhang, and I. Suemune, J. Appl. Phys. 98, 063502 (2005).

${ }^{13}$ S. Ganapathy, I. Suemune, P. Thilakan, H. Kumano, K. Uesugi, Y. Nabetani, T. Matsumoto, and H. Machida, Jpn. J. Appl. Phys., Part 2 44, L1512 (2005).

${ }^{14}$ J. D. Lambkin, D. J. Dunstan, K. P. Homewood, L. K. Howard, and M. T. Emeny, Appl. Phys. Lett. 57, 1986 (1990).

${ }^{15}$ J. W. Matthews and A. E. Blakeslee, J. Cryst. Growth 27, 118 (1974). 